\title{
Imaging morphologic substrates and molecular changes for differential diagnosis of vascular dementia
}

\author{
Wolf-Dieter Heiss ${ }^{1 *}$ \\ ${ }^{1}$ Max Planck Institute for Metabolism Research, Cologne, Germany
}

\begin{abstract}
The differential diagnosis of dementias is based on symptoms of cognitive and memory impairment and is supported by results of neuropsychological tests and of imaging. Whereas computed tomography and magnetic resonance imaging are able to detect morphologic substrates of vascular dementias as multi-infarct syndroms, large vessel and critically located strokes, small vessel disease and hemorrhages, these modalities cannot determine molecular causes and functional consequences of the underlying pathologies. Positron emission tomography allows imaging of the localized and/or diffuse metabolic and molecular disturbances responsible for cognitive impairment and dementia, and is effective in differentiating vascular from degenerative dementia, as Alzheimer's disease. Imaging of neurotransmitters and of pathologically accumulated proteins as well as of inflammation additionally yields insight into disease specific pathophysiology. Despite that the broad clinical application of PET is limited, this technology has a great impact on research in dementia.
\end{abstract}

\section{Introduction}

Vascular etiologies are the third most common cause of dementia ( 8 to $10 \%$ ), following $\mathrm{AD}(60$ to $70 \%)$, and dementia with Lewy bodies (DLB) (10 to $25 \%)$ [1], but these numbers vary considerably according to the different criteria used for VaD. Furthermore, it is evident from autopsy studies that many patients have mixed dementias, often vascular disease with other conditions [2] Old criteria for $\mathrm{VaD}$ only included multi-infarct dementia [3] or dementia resulting from the cumulative effects of several clinically significant strokes, but the current criteria consider multi-infarct dementia as only one of several subtypes of $\mathrm{VaD}$, including single-stroke dementia and small vessel disease. Vascular cognitive impairment is a complex multifactorial condition manifested in various clinical syndromes [4].

\section{Imaging of morphologic substrates}

Neuroimaging provides important information on neuroanatomical substrate of the disorder, plays an important role in the diagnosis and adds to prediction of $\mathrm{VaD}$. Most acute stroke patients undergo computed tomography (CT) brain imaging; thus studies using CT are representative of the whole clinical population. In clinical practice, CT is performed primarily to exclude haemorrhage and some stroke mimics (such as brain tumours), and can often demonstrate early signs of ischaemia (e.g. swelling, hypodensity and hyperdense vessels) and old stroke lesions.

The three main neuroimaging patterns in $\mathrm{VaD}$ are large vessels strokes (macroangiopathy, arteriosclerosis), small vessel disease (microangiopathy, arteriolosclerosis), and microhemorrhages. Single large territorial strokes, especially in the middle cerebral artery (MCA) territory of the dominant hemisphere, or multiple smaller strokes in bilateral anterior cerebral artery (ACA) or posterior cerebral artery (PCA) territories, cause dementia in $\sim 30 \%$ of stroke survivors [5]. Single smaller strokes can also cause significant cognitive dysfunction when occurring in particular locations, such as the watershed territories, including the bilateral superior frontal gyrus or bilateral orbitofrontal
(ACA/MCA), angular gyrus (ACA/MCA/PCA), temporo-occipital junction, and inferior temporal gyrus (MCA/PCA) [6].

Small vessel disease causes incomplete or complete infarcts in the white matter or in subcortical gray matter nuclei [7]. Lacunae must be differentiated from perivascular Virchow-Robin spaces. Lacunar strokes are small complete infarcts (2 to $15 \mathrm{~mm}$ ). When located in the caudate head, anterior thalamus, or the mamillothalamic tract [8] lacunae can cause significant cognitive and/or behavioral dysfunction due to the extended functional deafferentation of the cortical areas.

The presence and severity of white matter lesions (WMLs) and brain atrophy can be readily determined from CT brain scans - features which may predict subsequent cognitive impairment and dementia. There is very good agreement between brain atrophy and presence of moderate-severe white matter lesions on CT and MRI measures $[9,10]$.

Magnetic resonance imaging (MRI) remains the key neuroimaging modality in $\mathrm{VaD}$ [11]. If not contraindicated, MRI, rather than $\mathrm{CT}$, is preferred for research and routine clinical use because it has higher sensitivity and specificity for detecting pathological changes [12]. Standards for neuroimaging with a widely accepted terminology permitting comparison of findings between centers have been recommended (STandards for ReportIng Vascular changes on nEuroimaging, STRIVE) [13]. Numerous studies identified MRI markers of small vessel disease (SVD) (lacunes, white matter hyperintensities, cerebral microbleeds, silent infarcts, white matter changes, global cerebral atrophy, medial-temporal lobe atrophy) as determinants of $\mathrm{VaD}$. Vascular lesions traditionally attributed to $\mathrm{VaD}$ comprise subcortical areas of the brain, especially sub-frontal white matter circuits, strategic areas of single infarction such as the dominant

Correspondence to: Wolf-Dieter Heiss, Max Planck Institute for Metabolism Research, Cologne, Germany, E-mail: wdh@sf.mpg.de

Received: January 24, 2017; Accepted: February 20, 2017; Published: February 23,2017 
thalamus or angular gyrus, deep frontal areas and the left hemisphere, and bilateral brain infarcts or volume-driven cortical-subcortical infarctions reaching a critical threshold of tissue loss or injury [2].

Small vessel disease identified on MRI in the white matter is called leukoaraiosis [14]. Leukoaraiosis presents as multiple punctuate or confluent lesions, but more often as incomplete infarcts, and is commonly seen in healthy elderly and in subjects with migraine [15]. The markers of small vessel disease - white matter hyperintensities, lacunes, dilated vascular spaces, microbleeds, and brain volume - are related to decrease in regional cerebral blood flow [16] and must be clearly defined to be reliably used for the diagnosis of this vascular disorder and its progression [17]. Some studies have suggested that to assess in single cases how much the lesion load affects cognition, a threshold of $10 \mathrm{~cm}^{2}$ [15] or $25 \%$ of total white matter [18] is required before $\mathrm{VaD}$ is detectable clinically. On FLAIR images, incomplete infarcts present as hyperintensities, whereas complete infarcts present as lacunae, which are hypointense in relation to the brain and isointense to the cerebrospinal fluid. After stroke, medial temporal lobe atrophy is rather related to cognitive impairment than markers of small vessel disease [19]. When small vessel disease causes subcortical VaD, this is associated with the pathology of Binswanger's disease [20].

Microhemorrhages are the third major neuroimaging aspect of $\mathrm{VaD}$, and in one study they were found in $65 \%$ of $\mathrm{VaD}$ cases [21]. While macrohemorrhages associated with cognitive impairment (e.g., venous infarcts) can be seen on conventional T1- and T2-weighted spin echo images, microhemorrhages often cannot be seen in these sequences, but can be detected accurately using $\mathrm{T} 2^{*}$-weighted gradient echo images. In many cases, it is likely that microhemorrhages and white matter ischemic disease are caused by systemic hypertension [22].

\section{Molecular imaging in the diagnosis of vascular dementia}

The diagnosis of vascular cognitive impairment (VCI) is difficult because there is no consensus on clinical criteria. Additionally, cerebral arteriosclerosis frequently is present in elderly patients and even small infarcts or white matter lesions occur in elderly subjects without either cognitive impairment or degenerative dementia. There is a tendency to diagnose VCI on the basis of MRI, which has a high sensitivity for white matter hyperintensities (WMHs), which may be seen in normal elderly as well as those with VCI. Pathological studies reveal a high incidence of both vascular and degenerative pathology of the Alzheimer type. This leads to diagnostic confusion when only the MRI is used, and there is mixed pathologies. PET provides additional information, which increases the diagnostic certainty.

Positron emission tomography can support the clinical diagnosis by visualizing cerebral functions in typically affected brain regions. PET of ${ }^{18} \mathrm{~F}$-2-fluoro-2-deoxy-D-glucose (FDG) for measurement of regional cerebral glucose metabolism (rCMRGl) has shown a typical metabolic pattern in patients with probable $\mathrm{AD}$ : hypometabolism in temporoparietal and frontal association areas, but relative recessing of primary cortical areas, basal ganglia and cerebellum. In VCI a different pattern is seen [23]: In VCI FDG PET can clearly differentiate scattered areas of focal cortical and subcortical hypometabolism that differ from the typical metabolic pattern seen in $\mathrm{AD}$ with marked hypometabolism affecting the association areas [24]. In VCI patients [25] a significant reduction of rCMRglc in comparison to normal patients was observed in widespread cerebral regions (middle frontal cortex, temporoparietal cortex, basal ganglia, cerebellum and brainstem). In subcortical areas and primary sensorimotor cortex this hypometabolism was more marked than in $\mathrm{AD}$ while the association areas were less affected than in AD. A metabolic ratio (rCMRglc of association areas divided by rCMRglc of primary areas, basal ganglia, cerebellum and brainstem) mainly reflecting the contrast between association areas and subcortical regions was significantly lower in $\mathrm{AD}$ than that in VCI. Whereas it was not possible to identify a single region that could discriminate between $\mathrm{VCI}$ and $\mathrm{AD}$, the composite pattern, as expressed in the metabolic ratio, was significantly different. Considering that the VCI patients in that study had mainly WMHs and small subcortical infarcts, it suggests furthermore that even small infarcts in combination with WMHs may contribute to cognitive decline. Rather than the total volume of infarction, the volume of functional tissue loss is more important, since it also includes the effects of incompletely infarcted tissue and morphologically intact but deafferented cortex.

Few reports deal with the accuracy of rCMRglc changes with regard to the clinical diagnosis of AD compared with controls and other types of dementia. With the analysis of receiver operating characteristics (ROC) Herholz et al. [26] recorded $93 \%$ sensitivity at $83 \%$ specificity for differentiation of patients with probable $\mathrm{AD}$ from those without $\mathrm{AD}$ or other dementing illnesses. Subcortical ischemic vascular disease (SIVD) can be distinguished from clinically probable AD by a more diffuse pattern of hypometabolism involving also the primary cortices, basal ganglia, thalamus and cerebellum.

\section{Differential diagnosis of dementias with FDG-PET}

Alzheimer's disease (AD) is characterized by regional impairment of cerebral glucose metabolism in neocortical association areas, whereas the primary visual and sensorimotor cortex, basal ganglia, and cerebellum are relatively well preserved [27]. In a multicentre study comprising 10 PET centers that employed an automated voxel-based analysis of FDG PET images, the distinction between controls and AD patients had $93 \%$ sensitivity and $93 \%$ specificity [26]. Significantly abnormal metabolism in mild cognitive impairment (MCI) indicates a high risk to develop dementia within the next two years. Reduced neocortical glucose metabolism can probably be detected with FDG $\mathrm{PET}$ in $\mathrm{AD}$ on average one year before onset of subjective cognitive impairment.

Characteristic patterns of regional hypometabolism are also seen in other degenerative dementias [28]. Frontotemporal dementia (FTD) clinically characterized by changes in personality and behavior, semantic deficits and progressive aphasia can be identified by distinct frontal or frontotemporal metabolic impairments that are typically quite asymmetrically centered in the frontolateral cortex and the anterior pole of the temporal lobe. Dementia with Lewy bodies (LBD), combining fluctuating consciousness, Parkinsonian symptoms and impairment of visual perception including hallucinations, shows reduction of glucose metabolism in primary visual cortex in addition to that in posterior association areas. Other degenerative disorders show typical hypometabolism in the specifically affected brain structures: the putamen and cortex in corticobasal degeneration, the caudate nucleus in Huntington's chorea, the frontal cortex and midbrain in progressive supranuclear palsy and pons and cerebellum in olivopontocerebellar atrophy. It is also important to note that depressive disorders may mimic cognitive impairment; in these cases glucose metabolism does not show regional abnormalities. 


\section{Imaging synaptic transmission and accumulation of pathologic proteins}

Additional PET tracers can further support the diagnosis of a type of dementia and also yield information on the underlying pathophysiology: Tracers permit the study of selectively affected transmitter / receptor systems, e.g. the cholinergic system in $\mathrm{AD}$ - significant reduction of cholinergic activity in the cortex of $\mathrm{AD}$ patients and those with $\mathrm{MCI}$ and early conversion to AD [29] or the dopaminergic system in LBD [30] and the detection of pathogenetic depositions, e.g. amyloid and tau in $\mathrm{AD}$ [31] or inflammatory reactions with microglia activations as in VCI. Especially the imaging of accumulation of pathologic proteins is a recent strategy to differentiate degenerative dementias: Amyloid is a pathogenetic product in the development of $\mathrm{AD}$ and its accumulation is a key finding in this disease. Its accumulation can be imaged by $11 \mathrm{C}$ labeled Pittsburgh Compound B (PiB) [32] or by several newer $18 \mathrm{~F}$ labeled tracers [33]. Whereas only small amounts of amyloid can be detected in the white matter in normal aging [34], accumulation is visible in the frontal and temporo-parietal cortex in $\mathrm{AD}$ and MCI. However, also in $20-30 \%$ of aged persons without relevant cognitive impairment an increased accumulation of amyloid can be detected [35], and the grade of amyloid deposition as detected by PET is not related to the severity of cognitive impairment [36]. That means that amyloid might be deposited in the brain eventually long before cognitive impairment is recognized. Amyloid deposition in combination with neuroinflammation as expressed in microglia activation might play a role in the development of post-stroke dementia [37].

A more specific pathologic protein produced in $\mathrm{AD}$ is tau, and its deposition in the mesial temporal lobe is an early marker of $\mathrm{AD}$ or MCI [38] and the amount of tau detected in the cortex by selective PETtracers is related to the severity of cognitive impairment [39]. These PETtracers also detect the primary pathological substrate in other degenerative dementias (e.g. tau in FTD) [40] and permit the differentiation between $\mathrm{AD}$ and $\mathrm{VCI}$ and other degenerative dementias $[41,42]$.

\section{Conclusion}

The diagnosis of vascular dementia $(\mathrm{VaD})$ is difficult because there is no consensus on clinical criteria. Therefore, imaging is critical in diagnosis and treatment of dementias, particularly in $\mathrm{VaD}$ because of the ability to visualize ischemic and hemorrhagic injury to the gray and white matter. Most patients undergo brain imaging by computed tomography, which is able to detect ischemic strokes, hemorrhages and brain atrophy and may also indicate white matter changes. Magnetic resonance imaging remains the key neuroimaging modality and is preferred to $\mathrm{CT}$ for research and routine clinical use in vascular cognitive impairment (VCI) because it has higher sensitivity and specificity for detecting pathological changes. These modalities for imaging morphology permit to detect vascular lesions traditionally attributed to VCI in subcortical areas of the brain, single infarction or lacunes in strategic areas (thalamus or angular gyrus), or large corticalsubcortical lesions reaching a critical threshold of tissue loss. . Multiple punctuate or confluent lesions can be seen in the white matter by MRI and were called leukoaraiosis, which is often seen in healthy elderly and in subjects with migraine. Another major neuroimaging finding of small vessel disease in VCI are microhemorrhages. However, while computed tomography and magnetic resonance imaging are able to detect morphologic lesions, these modalities cannot determine functional consequences of the underlying pathological changes. Additionally, pathological studies reveal a high incidence of mixed dementias with both vascular and degenerative pathology of the
Alzheimer type . This leads to diagnostic confusion when only MRI is used.

Positron emission tomography can support the clinical diagnosis by visualizing cerebral functions in typically affected brain regions: In VaD FDG PET can clearly differentiate scattered areas of focal cortical and subcortical hypometabolism that differ from the typical metabolic pattern seen in AD with marked hypometabolism affecting the association areas. Additional PET tracers can further support the diagnosis of a type of dementia and also yield information on the underlying pathophysiology: Tracers permit the study of selectively affected transmitter / receptor systems, e.g. the cholinergic system in $\mathrm{AD}$. The imaging of accumulation of pathologic proteins is a recent strategy to differentiate degenerative and vascular dementias: Amyloid is involved in pathogenesis of $\mathrm{AD}$ and its accumulation is a key finding in this disease. An even more specific pathologic protein is tau, and its deposition in various brain structures is an early marker correlated to cognitive impairment in several degenerative dementias. As these studies provide insight in early changes of the diseases, selective PETstudies might be useful to detect preclinical stages in which therapeutic efforts might be promising. Multimodal imaging including advanced techniques for morphological and molecular imaging are important for research in dementias, but are also more and more introduced into clinical application [43-45].

\section{References}

1. Zylak CJ, Dyck DR, Warren P, Tse KS (1975) Hypersensitive lung disease due to avian antigens. Radiology 114: 45-49. [Crossref]

2. Jellinger KA (2007) The enigma of vascular cognitive disorder and vascular dementia. Acta Neuropathol 113: 349-388. [Crossref]

3. Hachinski VC, Iliff LD, Zilhka E, Du Boulay GH, McAllister VL, et al. (1975) Cerebral blood flow in dementia. Arch Neurol 32: 632-637. [Crossref]

4. Iadecola C (2013) The pathobiology of vascular dementia. Neuron 80: 844-866 [Crossref]

5. Guermazi A, Miaux Y, Rovira-Cañellas A, Suhy J, Pauls J, et al. (2007) Neuroradiological findings in vascular dementia. Neuroradiology 49: 1-22. [Crossref]

6. Pohjasvaara T, Mäntylä R, Salonen O, Aronen HJ, Ylikoski R, et al. (2000) MRI correlates of dementia after first clinical ischemic stroke. J Neurol Sci 181: 111-117. [Crossref]

7. Roman GC, Erkinjuntti T, Wallin A, Pantoni L, Chui HC (2002) Subcortical ischaemic vascular dementia. Lancet Neurology 1: 426-436. [Crossref]

8. Carrera E, Bogousslavsky J (2006) The thalamus and behavior: effects of anatomically distinct strokes. Neurology 66: 1817-1823. [Crossref]

9. Wattjes MP, Henneman WJ, van der Flier WM, Oscar de Vries, Frank Träber, et al (2009) Diagnostic imaging of patients in a memory clinic: comparison of MR imaging and 64-detector row CT. Radiology 253: 174-183. [Crossref]

10. Wahlund LO, Barkhof F, Fazekas F, Bronge L, Augustin M, et al. (2001) A new rating scale for age-related white matter changes applicable to MRI and CT. Stroke 32: 13181322. [Crossref]

11. Vitali P, Migliaccio R, Agosta F, Rosen HJ, Geschwind MD (2008) Neuroimaging in dementia. Semin Neurol 28: 467-483. [Crossref]

12. Brainin M, Tuomilehto J, Heiss WD, Bornstein NM, Bath PM, et al. (2015) Post-stroke cognitive decline: an update and perspectives for clinical research. Eur J Neurol 22: 229-238, e13-6. [Crossref]

13. Wardlaw JM, Smith EE, Biessels GJ, Cordonnier C, Fazekas F, et al. (2013) Neuroimaging standards for research into small vessel disease and its contribution to ageing and neurodegeneration. Lancet Neurol 12: 822-838. [Crossref]

14. Hachinski VC, Potter P, Merskey H (1987) Leuko-araiosis. Arch Neurol 44: 21-23. [Crossref]

15. Boone KB, Miller BL, Lesser IM, et al. (1992) Neuropsychological correlates of whitematter lesions in healthy elderly subjects. A threshold effect. Arch Neurol 49: 549-554 [Crossref] 
16. Shi Y, Thrippleton MJ, Makin SD, Marshall I, Geerlings MI, et al. (2016) Cerebral blood flow in small vessel disease: A systematic review and meta-analysis. $J$ Cereb Blood Flow Metab 36: 1653-1667. [Crossref]

17. De Guio F, Jouvent E, Biessels GJ, Sandra E Black,Carol Brayne, et al. (2016) Reproducibility and variability of quantitative magnetic resonance imaging markers in cerebral small vessel disease. J Cereb Blood Flow Metab 36: 1319-1337. [Crossref]

18. van Straaten EC, Scheltens P, Knol DL, van Buchem MA, van Dijk EJ, et al. (2003) Operational definitions for the NINDS-AIREN criteria for vascular dementia: an interobserver study. Stroke 34: 1907-1912. [Crossref]

19. Arba F, Quinn T, Hankey GJ, Ali M, Lees KR, et al. (2017) Cerebral small vessel disease, medial temporal lobe atrophy and cognitive status in patients with ischaemic stroke and transient ischaemic attack. Eur J Neurol 24: 276-282. [Crossref]

20. Román GC (1987) Senile dementia of the Binswanger type. A vascular form of dementia in the elderly. JAMA 258: 1782-1788. [Crossref]

21. Cordonnier C, van der Flier WM, Sluimer JD, Leys D, Barkhof F, et al. (2006) Prevalence and severity of microbleeds in a memory clinic setting. Neurology 66: 13561360. [Crossref]

22. Koennecke HC (2006) Cerebral microbleeds on MRI: prevalence, associations, and potential clinical implications. Neurology 66: 165-171. [Crossref]

23. Heiss WD, Zimmermann-Meinzingen S (2012) PET imaging in the differential diagnosis of vascular dementia. J Neurol Sci 322: 268-273. [Crossref]

24. Benson DF, Kuhl DE, Hawkins RA, Phelps ME, Cummings JL, et al. (1983) The fluorodeoxyglucose 18F scan in Alzheimer's disease and multi-infarct dementia. Arch Neurol 40: 711-714. [Crossref]

25. Mielke R, Herholz K, Grond M, Kessler J, Heiss WD (1992) Severity of vascular dementia is related to volume of metabolically impaired tissue. Arch Neurol 49: 909913. [Crossref]

26. Herholz K, Salmon E, Perani D, Baron JC, Holthoff V, et al. (2002) Discrimination between Alzheimer dementia and controls by automated analysis of multicenter FDG PET. Neuroimage 17: 302-316. [Crossref]

27. Herholz K (2003) PET studies in dementia. Ann Nucl Med 17: 79-89. [Crossref]

28. Bohnen NI, Djang DS, Herholz K, Anzai Y, Minoshima S (2012) Effectiveness and safety of $18 \mathrm{~F}-\mathrm{FDG}$ PET in the evaluation of dementia: a review of the recent literature. $J$ Nucl Med 53: 59-71. [Crossref]

29. Herholz K, Weisenbach S, Kalbe E, Diederich NJ, Heiss WD (2005) Cerebral acetylcholine esterase activity in mild cognitive impairment. Neuroreport 16: 14311434. [Crossref]

30. Hilker R, Thomas AV, Klein JC, Weisenbach S, Kalbe E, et al. (2005) Dementia in Parkinson disease: functional imaging of cholinergic and dopaminergic pathways. Neurology 65: 1716-1722. [Crossref]
31. Braak H, Braak E (1991) Neuropathological stageing of Alzheimer-related changes. Acta Neuropathol 82: 239-259. [Crossref]

32. Klunk WE, Engler H, Nordberg A, Wang Y, Blomqvist G, et al. (2004) Imaging brain amyloid in Alzheimer's disease with Pittsburgh Compound-B. Ann Neurol 55: 306-319. [Crossref]

33. Villemagne VL, Mulligan RS, Pejoska S, Ong K, Jones G, et al. (2012) Comparison of $11 \mathrm{C}-\mathrm{PiB}$ and $18 \mathrm{~F}$-florbetaben for $\mathrm{A}^{2}$ imaging in ageing and Alzheimer's disease. Eur J Nucl Med Mol Imaging 39: 983-989. [Crossref]

34. Aizenstein HJ, Nebes RD, Saxton JA, Price JC, Mathis CA, et al. (2008) Frequent amyloid deposition without significant cognitive impairment among the elderly. Arch Neurol 65: 1509-1517. [Crossref]

35. Herholz K, Ebmeier K (2011) Clinical amyloid imaging in Alzheimer's disease. Lancet Neurol 10: 667-670. [Crossref]

36. Yotten RA, Doshi J, Clark V, Jitka Sojkova, Yun Zhou, et al. (2013) Memory decline shows stronger associations with estimated spatial patterns of amyloid deposition progression than total amyloid burden. Neurobiology of aging 34: 2835-2842. [Crossref]

37. Thiel A, Cechetto DF, Heiss WD, Hachinski V, Whitehead SN (2014) Amyloid burden, neuroinflammation, and links to cognitive decline after ischemic stroke. Stroke 45: 2825-2829. [Crossref]

38. Maruyama M, Shimada H, Suhara T, Shinotoh H, Ji B, et al. (2013) Imaging of tau pathology in a tauopathy mouse model and in Alzheimer patients compared to normal controls. Neuron 79: 1094-1108. [Crossref]

39. Small GW, Bookheimer SY, Thompson PM, Cole GM, Huang SC, et al. (2008) Current and future uses of neuroimaging for cognitively impaired patients. Lancet Neurol 7: 161-172. [Crossref]

40. Spillantini MG, Goedert M (2013) Tau pathology and neurodegeneration. Lancet Neurol 12: 609-622. [Crossref]

41. Schöll M, Lockhart SN, Schonhaut DR, O’Neil JP, Janabi M, et al. (2016) PET Imaging of Tau Deposition in the Aging Human Brain. Neuron 89: 971-982. [Crossref]

42. Sepulcre J, Schultz AP, Sabuncu M, Gomez-Isla T, Chhatwal J, et al. (2016) In Vivo Tau, Amyloid, and Gray Matter Profiles in the Aging Brain. J Neurosci 36: 7364-7374. [Crossref]

43. Banerjee G, Wilson D, Jager HR, Werring DJ (2016) Novel imaging techniques in cerebral small vessel diseases and vascular cognitive impairment. Biochim Biophys Acta 1862: 926-938. [Crossref]

44. Narayanan L, Murray AD (2016) What can imaging tell us about cognitive impairment and dementia? World J Radiol 8: 240-254. [Crossref]

45. Heiss WD, Rosenberg GA, Thiel A, Berlot R, de Reuck J (2016) Neuroimaging in vascular cognitive impairment: a state-of-the-art review. BMC Med 14: 174. [Crossref]

Copyright: $\odot 2017$ Heiss WD. This is an open-access article distributed under the terms of the Creative Commons Attribution License, which permits unrestricted use, distribution, and reproduction in any medium, provided the original author and source are credited. 